\title{
Performance of the large-scale drift chamber setup in cosmic ray experiment
}

\author{
A.G. Bogdanov ${ }^{1, a}$, N.V. Ampilogov ${ }^{a}$, N.S. Barbashina ${ }^{a}$, A.A. Borisov ${ }^{a, b}$, \\ R.M. Fakhrutdinov ${ }^{a, b}$, R.P. Kokoulin ${ }^{a}$, K.G. Kompaniets ${ }^{a}$, A.S. Kozhin ${ }^{a, b}$,
} A.S. Ovechkin ${ }^{a}$, A.A. Petrukhin ${ }^{a}$, N.S. Volkov ${ }^{a}$, V.S. Vorobyev ${ }^{a}$, I.I. Yashin ${ }^{a}$, E.A. Zadeba ${ }^{a}$

${ }^{a}$ National Research Nuclear University MEPhI (Moscow Engineering Physics Institute), 115409

Moscow, Russian Federation

${ }^{b}$ RF SSC Institute of High Energy Physics, 142281, Protvino, Russian Federation

E-mail: AGBogdanov@mephi.ru

\begin{abstract}
The new coordinate-tracking unit on drift chambers (CTUDC) is launched in MEPhI. The detector is developed for joint operation with Cherenkov water detector (CWD) NEVOD of 2000 cubic meters and coordinate-tracking detector DECOR. It represents two coordinate planes of 8 large multi-wire drift chambers in each mounted on the opposite sides of CWD. The total area of the detector is about 30 square meters. The aim of the project is to examine the features of joint operation of such type detectors with NEVOD triggering system, accuracy and performance of drift chambers. This information will be used in development of a future largescale detector TREK consisting of 264 same drift chambers. Results of cross-calibration of the CTUDC and coordinate-tracking detector DECOR, joint registration of high-multiplicity muon bundles with NEVOD-DECOR complex and performance of the new setup are presented.
\end{abstract}

35th International Cosmic Ray Conference - ICRC2017

10-20 July, 2017

Bexco, Busan, Korea

${ }^{1}$ Speaker 


\section{Introduction}

The aim of the large-scale coordinate-tracking detector TREK construction is solving the problem of the excess of muon bundles that increases with the energy of the primary cosmic rays $[1,2]$, what can be caused by both cosmo- or nuclear-physical reasons [3]. The only characteristics that responds differently to changes in the composition of cosmic rays and the inclusion of new physical processes is the energy of the muon component of extensive air showers [3] which has not been investigated to the present time. Such studies are performed at the experimental complex NEVOD-DECOR $[4,5]$; however, the coordinate detector DECOR does not cover the entire aperture of the Cherenkov water detector and does not exclude the possibility of passing of several muons between the individual supermodules of the detector. Besides, the size of its cells limits the possibility of separating two or more particles at small distances (less than $3 \mathrm{~cm}$ ). The new coordinate-tracking detector [6] based on drift chambers will increase the coverage of the side aperture of the Cherenkov water detector (CWD) NEVOD and significantly improve the resolution of close tracks.

\section{Drift chambers}

Drift chamber was developed specially for the neutrino experiment at IHEP [7]. The specific features of such experiments (low event rate, moderate requirements to spatial resolution) allow the use of drift chambers with a large drift gap for detection of particle tracks.

Overall sizes of the chambers are $4000 \times 508 \times 112 \mathrm{~mm}^{3}$. Sensitive area is $1.85 \mathrm{~m}^{2}$ that is $91 \%$ of the chamber area. The chamber represents an aluminum box with ends limited by Plexiglas plugs, to which wires, gas inlets and high voltage connectors are mounted. The drift chamber is filled with a gas mixture of $94 \% \mathrm{Ar}$ and $6 \% \mathrm{CO}_{2}$. A uniform electric field is formed by field-forming wires with a pitch of $5 \mathrm{~mm}$, the potential on them varies linearly from 0 to 12 $\mathrm{kV}$. The drift chamber has four signal wires alternately shifted by $\pm 0.75 \mathrm{~mm}$ in the drift direction relative to the center of the chamber; such configuration allows reconstructing the projection of the track on the plane orthogonal to the wires. Thus, for the reconstruction of tracks in space at least two non-parallel drift chambers are required. Spatial accuracy of the chamber is $1 \mathrm{~mm}$, the angular resolution is about $1.5^{\circ}$, two close tracks can be separated at a distance of $3 \mathrm{~mm}$.

The signals from the wires are processed by an on-board amplifier-shaper that forms 75$100 \mathrm{~ns}$ pulses (depending on the input signal) in the LVDS levels.

The three decades passed since drift chambers were produced in IHEP, so before assembling in the new setup they needed attentive examination. The first test of the chambers was held at IHEP after dismatling of the neutrino experimental setup. All chambers were checked for leaks and wire integrity at high voltage. After that the first batch (32 chambers) were delivered to MEPhI. Two test benches were used for their study. The first one, representing multi-level frame construction with scintillator muon telescope [8], allowed investigating noise characteristics and roughly coordinate and angular resolution.

Further study of the drift chambers in the flux of cosmic rays was conducted with the supermodule (SM) of the muon hodoscope URAGAN. The testing technique is based on coregistration of single muons at the studied detector and at the muon hodoscope that provides high spatial accuracy of the reconstructed track of the muon $[9,10]$. As the result, the efficiency, coordinate and angular accuracy of drift chambers were investigated with high precision, also the spatial distributions of these characteristics across the active area of the chamber were 
obtained. The chambers demonstrated exellent performance, showed angular accuracy better than $1.2^{\circ}$ and coordinate resolution better than $1 \mathrm{~mm}$.

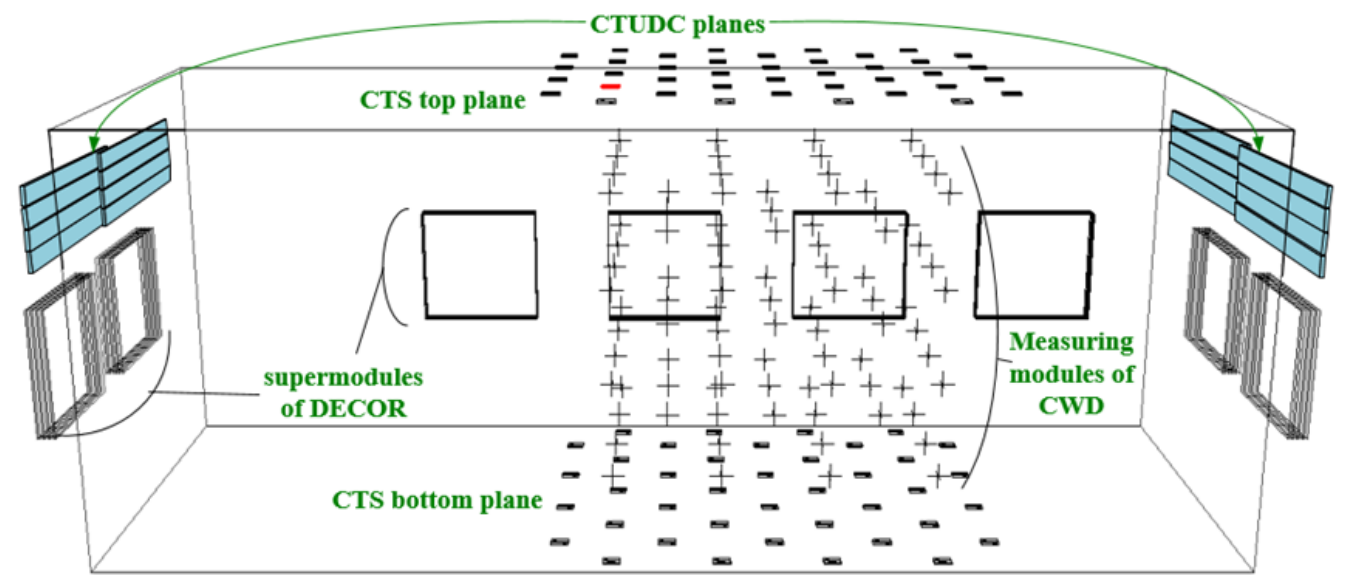

Figure 1: Arrangement of the CTUDC planes relative to DECOR and CWD.

\section{Coordinate-Tracking Unit on Drift Chambers (CTUDC)}

Coordinate-tracking unit based on drift chambers (CTUDC) consists of two vertical coordinate planes installed at the opposite sides of the CWD in the short galleries of the third floor of the NEVOD building, one floor above the DECOR supermodules (Fig. 1). Such location allows the registration of near-horizontal tracks as by CTUDC (triggered by CWD) so by joint operation with DECOR.

Each plane consists of 8 drift chambers installed in two rows, overlapped by $30 \mathrm{~cm}$ to exclude dead zones in the chamber ends; it causes a $4^{\circ}$ angle between the planes and CWD wall. Chambers are mounted on a special frame that allows precise adjustment in all directions.

CTUDC is designed for joint operation with the triggering system of the experimental complex NEVOD (NEVOD TS) that binds registration systems of CWD, DECOR, calibration telescope system (CTS) [11] and array of neutron detectors PRISMA. Triggering system has a rather fast data handling: the interval between the passage of a particle through the working volume of CWD and the trigger formation is about $500 \mathrm{~ns}$. On the other hand, the maximum drift time of electrons in the drift chamber is $6 \mu \mathrm{s}$, so registration system and DAQ of CTUDC cannot be directly integrated into the NEVOD TS and should be implemented separately with possibility of off-line inter-connection between the NEVOD and CTUDC data.

The registration system of CTUDC consists of a CTUDC main PC (MPC), VME crate with an optical bridge and a 128-channel time-to-digital converter (TDC) CAEN V1190 [12]. The TDC resolution is $100 \mathrm{ps}$ while the time resolution of the drift chambers is about $5 \mathrm{~ns}$. The internal memory of the device allows storing large amount of events to the moment of TDC readout.

The NEVOD TS fixes the event if one of the experimental complex setups is triggered: CWD, DECOR, CTS or PRISMA. Each setup has its own trigger conditions. In the main mode of operation, trigger for CAEN TDC should be received after all hits of the event, so the signal from NEVOD TS is delayed by $8 \mu \mathrm{s}$. The matching window of TDC is chosen to be certainly longer than the delay value, so it is $12 \mu \mathrm{s}$. 
For several types of events, MPC of NEVOD sends a network packet via Ethernet with information about the last event for further matching of CTUDC and NEVOD TS data, the most important information in the packet is the event number. This information is used for event matching between CTUDC and NEVOD-DECOR.
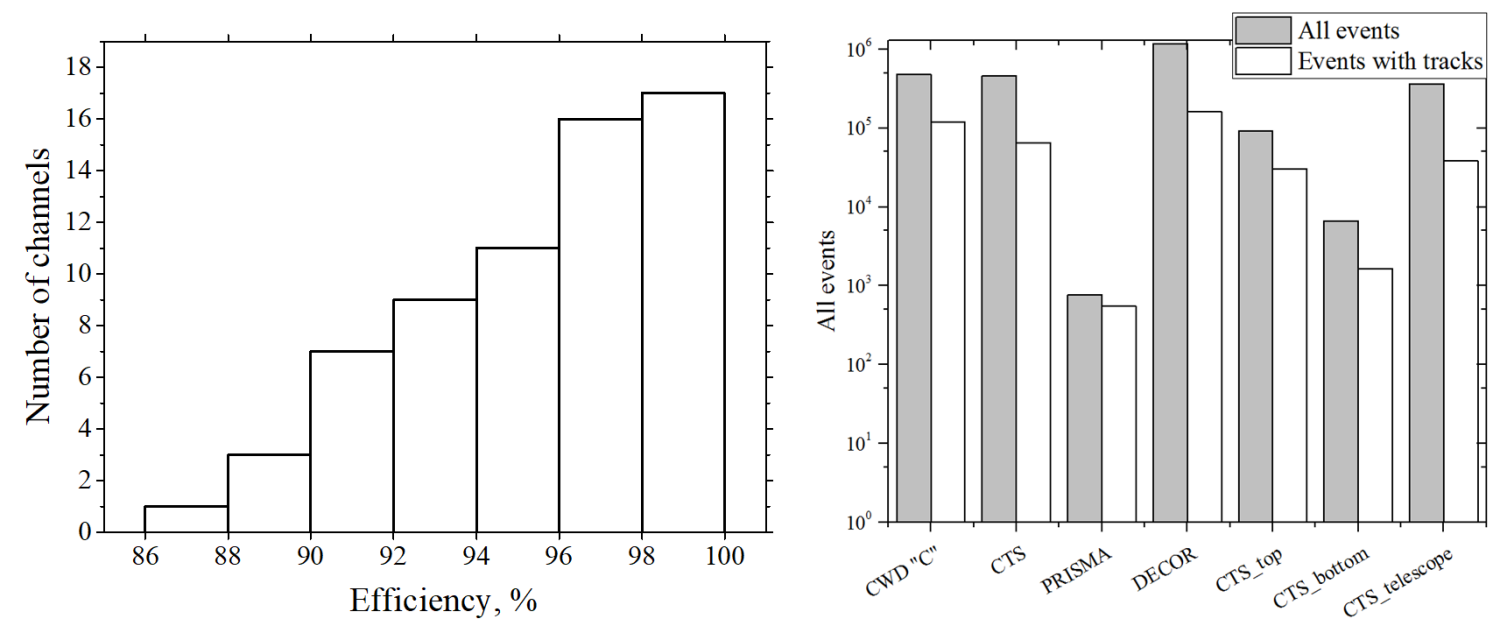

Figure 2: Efficiency of CTUDC measuring channels (left) and the number of events per each trigger for one experimental run (right).

\section{Joint operation of CTUDC with NEVOD-DECOR}

For the moment, more than 3000 hours of debugging and measuring series held at the CTUDC. Drift chambers showed a good performance, mean efficiency of CTUDC measuring chambers is $95.5 \%$ (Figure 2, left), that is better than at the neutrino experiment in IHEP (93\%), but lower than at the test benches. The right plot in Figure 2 shows the distribution of events according to the setups that gave trigger to NEVOD TS. The graph shows that the least likely response in CTUDC is accompanied by events from CTS and DECOR (usually these are single muon events). Most of events from the PRISMA are usually accompanied by CTUDC response.

According to their data, a cross-calibration with the coordinate-tracking detector DECOR was made and calibrations of TDC offsets and drift velocities were conducted.
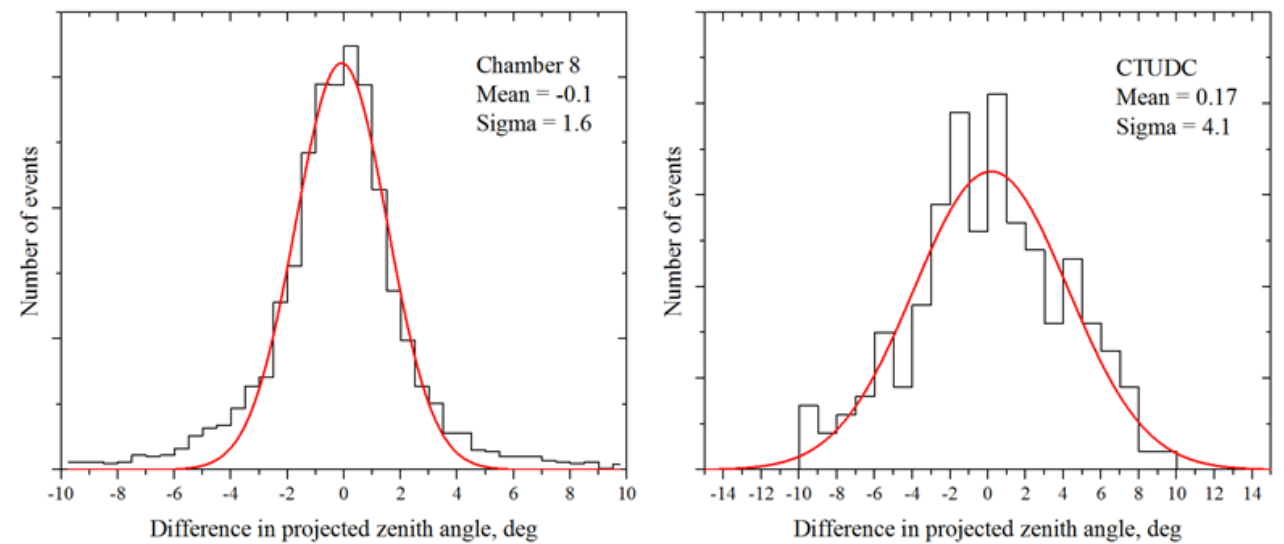

Figure 3: Distribution of events in difference in projected zenith angles estimated by CTUDC and DECOR data for single muons (left), for muon bundles (right). 
In experimental data, events containing tracks in DECOR and CTUDC were chosen. Than for each drift chamber a comparison similar to tests at the URAGAN supermodule was made. The only differences are relatively small statistics and long distances between the chambers and DECOR supermodules.

The left plot in Figure 3 shows the distribution of events in the difference in the muon track projected zenith angle estimated according to CTUDC and DECOR reconstruction. The form of the distribution is barely non-gaussian because the different types of tracks are 0included in statistics and all of them pass through the chamber in the same direction. Nearhorizontal tracks corresponding to high energy muons have a rather low scattering at their way through the water and concrete walls, so the difference in angle between CWD and DECOR for such tracks is smaller. Muons at lower angles have lower energy, so their scattering is higher. Besides that, the standard deviation for this distribution is $2.4^{\circ}$ that shows a good agreement between two setups.

This comparison of tracks allowed to make calibration of CTUDC. Distributions of zenith angles and coordinates gave the corrections for drift chamber alignment, it is especially important for muon bundles investigation, because zenith angle is one of their two main characteristics, along with the multiplicity.

The next study was taken for muon bundles registration in CTUDC and DECOR. Each multi-particle event was reconstructed separately with these setups, only shielded with water tank supermodules and drift chambers were considered from event data. The right plot in Figure 3 shows the distribution of events in the difference in the projected zenith angles of muon bundles in these events, the standard deviation is $4^{\circ}$. It means that some further improvements of reconstruction methods are needed.

Figure 4 shows an example of a muon bundle registered in CTUDC. The event data are processed with histogram method; it means that only near-parallel tracks can be reconstructed. Time series for each drift chambers are presented in the bottom part of the Figure 5, some nonparallel tracks from secondary particles can be seen there. The projected zenith angle of a bundle is $65^{\circ}$ for both planes that is close to DECOR data $\left(68^{\circ}\right)$, the total number of reconstructed tracks is 32 .

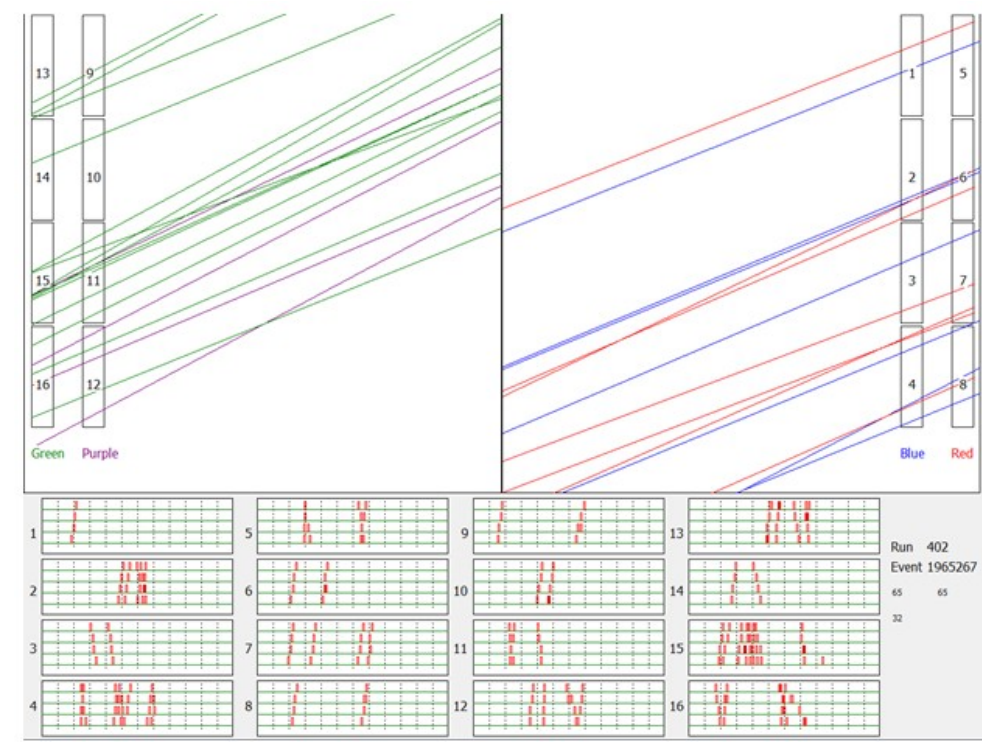

Figure 4: An example of a muon bundle registered by CTUDC. 


\section{Registration of high multiplicity muon bundles}

The reconstruction of multi-particle events in CTUDC is performed with several methods. The main is a method of a histogram [13]. The point of muon generation is rather distant from the detector so muon tracks are quasi-parallel. The method finds and reconstructs parallel tracks in all drift chambers in both planes, it rejects most part of secondary particles and selects muons. Figure 5 shows the distribution of events according to the number of reconstructed tracks in them, it almost fits with power function with -1.9 index. The maximum number of tracks in a single chamber is 29 .

The response of the detector is very sensitive to the azimuth angle of the bundle. The best performance can be reached when muon tracks are almost in the plane perpendicular to drift chambers. On the other side, horizontal muons give the most clear tracks in the detector. The current configuration of the detector allows only to measure projected zenith angle, so the azimuth can be taken from DECOR data. It is also necessary for estimations of the muon density since the detector effective area for each direction is different. The future TREK setup will measure both projections of muon tracks, its planes will have orthogonal orientation of the drift chambers.

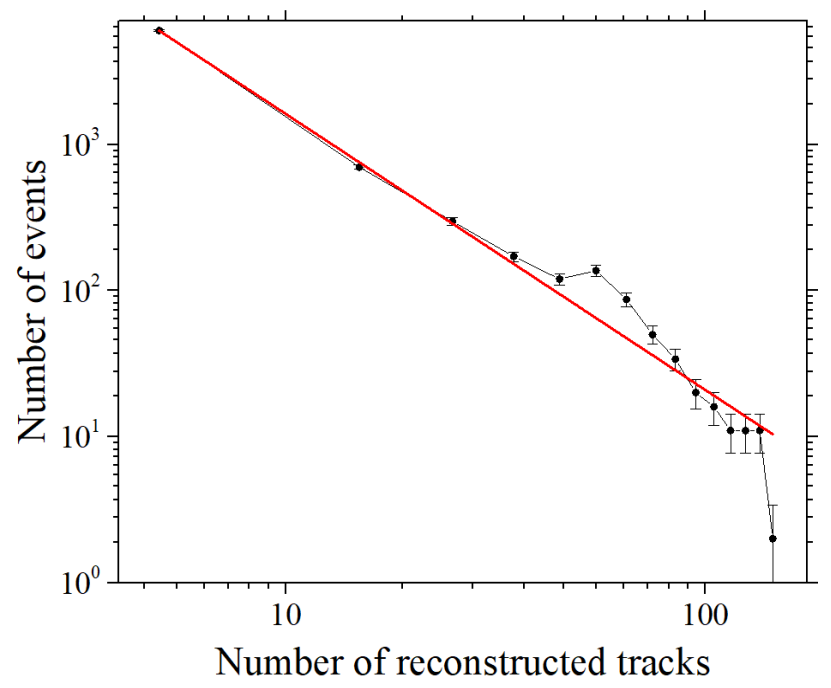

Figure 5: The distribution of events according to the number of reconstructed tracks.

\section{Conclusion}

The new coordinate-tracking unit CTUDC has been created in MEPhI; it represents the prototype of the future large-scale detector TREK. The unit showed good performance, multiwire drift chambers from IHEP proved their durability, high efficiency and good resolution. The registration system of CTUDC has the same principle of joint operation with NEVOD-DECOR triggering system so further it will be expanded to 1120 channels for a full-scale detector.

\section{Acknowledgments}

This work was performed at the Unique Scientific Facility "Experimental complex NEVOD" with the support of the Ministry of Education and Science of the Russian Federation 
(contract RFMEFI59114X0002 and MEPhI Academic Excellence Project of August 27, 2013, no. 02.a03.21.0005) and the Russian Foundation for Basic Research (grant 16-29-13028-ofi-m).

\section{References}

[1] N.S. Barbashina et al., Ultra-high energy cosmic ray investigations by means of EAS muon density measurements. Nucl. Phys. B (Proc. Suppl.). 165 (2007) 317.

[2] A.G. Bogdanov et al., Investigation of the properties of the flux and interaction of ultrahigh-energy cosmic rays by the method of local-muon-density spectra. Physics of Atomic Nuclei 73 (2010) 1852.

[3] A.A. Petrukhin. NEVOD-DECOR experiment and evidences for quark-gluon plasma in cosmic rays. Nucl. Instrum. Methods Phys. Res. A 692 (2012) 228.

[4] O. Saavedra et al., NEVOD-DECOR experiment: results and future. J. Phys. Conf. Ser. 409 (2013) 012009 .

[5] A. A. Petrukhin, Cherenkov water detector NEVOD, Physics-Uspekhi 58 (2015) 486.

[6] E.A. Zadeba et al., The coordinate-tracking detector based on the drift chambers for ultrahighenergy cosmic ray investigations. J. Instrum. 9 (2014) C08018.

[7] N.I. Bozhko et al., Drift chamber for the Serpukhov neutrino detector. Nucl. Instr. Meth. Phys. Res. A 243 (1986) 388.

[8] E.A. Zadeba et al., Status of a development of the large scale coordinate-tracking setup based on the drift chambers. J. Phys. Conf. Ser. 632 (2015) 012031.

[9] N.S. Barbashina et al., The URAGAN wide-aperture large-area muon hodoscope. Instrum. Experim. Techniques. 51 (2008) 180.

[10] N. V. Ampilogov et al., Application of the URAGAN muon hodoscope to calibrate charged particle detectors, Bull. Russ. Acad. Sci. Phys. 79 (2015) 386.

[11] M. B. Amelchakov et al., Calibration telescope system of CWD NEVOD as a detector of electron and muon components of EAS, Phys. Procedia. 74 (2015) 449.

[12] E.A. Zadeba et al., The registration system of the coordinate-tracking setup on the drift chambers. $J$. Phys. Conf. Ser. 675 (2016) 032039.

[13] E.A. Zadeba et al., Registration of muon bundles by a coordinate tracking unit based on drift chambers, Bull. Russ. Acad. Sci. Phys. 81 (2017) 481. 